\title{
Down-Regulation of Renal Endothelial Nitric Oxide Synthase Expression in Experimental Glomerular Thrombotic Microangiopathy
}

\author{
Xin J. Zhou, Zoltan Laszik, Zhenmin Ni, Xiu Q. Wang, Daniel J. Brackett, \\ Megan R. Lerner, Fred G. Silva, and Nosratola D. Vaziri \\ Department of Pathology (XJZ), University of Texas Southwestern Medical Center, Dallas, Texas; Departments of \\ Pathology (ZL, FGS) and Surgery (DJB, MRL), University of Oklahoma Health Sciences Center, Oklahoma City, \\ Oklahoma; and Department of Medicine (ZN, XQW, NDV), University of California at Irvine, Irvine, California
}

\begin{abstract}
SUMMARY: Infection with certain strains of Escherichia coli and endotoxemia results in renal glomerular thrombotic microangiopathy (TMA) characterized by endothelial swelling and prominent glomerular microthrombus formation. Nitric oxide (NO) is an endogenous biologic modulator with diverse physiologic functions including vasodilation and inhibition of platelet adhesion and aggregation. NO is synthesized from conversion of L-arginine to L-citrulline by a family of NO synthases (NOS), which include constitutive and inducible isoforms. Indirect evidence supports the hypothesis that TMA is associated with depressed intrarenal NO production. However, the effect of TMA on renal tissue NOS expression has not been fully elucidated. We studied rats with TMA induced by iv bolus injection of high dose $(20 \mathrm{mg} / \mathrm{kg}) E$. coli endotoxin. Subgroups of six animals each were sacrificed before or at $30,90,180,360$, and 720 minutes after the administration of endotoxin. Renal histology and tissue expression of endothelial and inducible nitric oxide synthases (eNOS and iNOS) were examined. Additionally, we examined the effect of endotoxin on glomerular NO production, and eNOS and iNOS protein expression in vitro. Glomerular capillary thrombosis developed by 180 minutes after endotoxin administration in approximately half of the animals. The glomeruli without thrombotic lesions apparent by light microscopy disclosed early signs of TMA characterized by endothelial swelling, platelet accumulation/adhesion, and patchy fibrinogen deposition. These morphologic changes were associated with a marked reduction of renal tissue eNOS expression beyond 180 minutes after the endotoxin administration. The fall in eNOS expression was coupled with a significant rise in iNOS protein abundance, which was expressed largely by glomerular circulating neutrophils and endothelial cells, peritubular vascular endothelium, and collecting ducts of cortex and medulla. In vitro incubation of isolated glomeruli with endotoxin also resulted in a marked reduction in eNOS expression and a significant rise in iNOS content. Administration of $E$. coli endotoxin leads to a sustained fall in renal eNOS expression both in vivo and in vitro. The associated decline in intrarenal endothelial NO production/availability may result in renal vasoconstriction and a hypercoagulative state, which may contribute to the pathogenesis of endotoxin-induced TMA. (Lab Invest 2000, 80:1079-1087).
\end{abstract}

\begin{abstract}
$T$ nfection with certain strains of Escherichia coli and 1 endotoxemia lead to a number of functional and structural alterations of the kidney, including rapid deterioration of renal function, which has been characterized as a glomerular thrombotic microangiopathy (TMA) (Laszik et al, 1994a, 1994b). Some of these changes are caused by the direct effect of endotoxin and/or are mediated by endotoxin-induced proinflammatory cytokines such as interleukin-1 (IL-1) and tumor necrosis factor (TNF) (Bertani et al, 1989; Cybulsky et al, 1988; Wakabayashi et al, 1991). For example, Laszik et al have demonstrated a transient increase in IL- $1 \alpha$ but not TNF $\alpha$ gene products in glomerular endothelial cells in endotoxin- and E. coliinduced TMA (Laszik et al, 1994b). Endothelial activation by endotoxin and proinflammatory cytokines can
\end{abstract}

Received March 14, 2000.

Address reprint requests to: Dr. X. J. Zhou, Department of Pathology, University of Texas Southwestern Medical Center, 5323 Harry Hines Boulevard, Dallas, TX 75235-9073. Fax: (214) 590-1411; E-mail: jzhou@mednet.swmed.edu alter the balance of endothelial procoagulant/anticoagulant activity leading to clot formation (Bevilacqua et al, 1986; Nawroth et al, 1986; Nawroth and Stern, 1986; Pober and Cotran, 1991).

Nitric oxide (NO) is an important endogenous biologic modulator with diverse physiologic actions. These actions include vasodilation, inhibition of platelet adhesion/aggregation, suppression of cellular proliferation, and matrix production (Marletta, 1989; Moncada and Higgs, 1995). NO is synthesized from conversion of L-arginine to L-citrulline by a family of NO synthases (NOS), which include constitutive and inducible isoforms (Moncada et al, 1991). Basal release of NO by endothelial cells contributes to the maintenance of normal vasodilatory tone and thromboresistance (Radomski et al, 1987, 1993 ). Previously, Shultz and Raij (1992) demonstrated that rats given a low dose of endotoxin exhibit a marked increase in the urinary excretion of NO metabolites and no evidence of glomerular thrombosis. They also showed that prior or concomitant treatment with a nonselective NOS inhibitor leads to generalized glo- 
merular thrombosis in their model. The authors concluded that enhanced renal NO formation may play an important role in maintaining renal perfusion and preventing platelet aggregation and glomerular thrombosis in septic shock. Subsequently, the same group of investigators (Westberg et al, 1994) demonstrated that nitroglycerin, an exogenous NO donor, can prevent endotoxin-induced glomerular thrombosis in rats. Additionally, rats are more susceptible to endotoxininduced glomerular thrombosis during pregnancy, which is naturally associated with a decreased L-arginine level (Raij, 1994). These observations lend further support to the hypothesis that TMA is associated with depressed NO production and/or availability in the local renal microenvironment. However, several studies (Herlitz et al, 1997; Noris et al, 1996) in human subjects conclude that TMA is associated with increased NO formation manifested by increased urinary and plasma NO metabolites. Because regional NO production in the kidney was not investigated in the previous animal and human studies, we investigated the effect of TMA on the expression of endothelial and inducible NOS (eNOS and iNOS) in the kidneys in rats with high dose endotoxin-induced TMA. Additionally, we examined the effect of endotoxin on glomerular NO production and eNOS and iNOS protein expression in vitro.

\section{Results}

\section{Light Microscopy and Direct Immunofluorescence (IF)}

By light microscopy, three of the six endotoxin-treated animals showed extensive glomerular thrombosis at 180 and 360 minutes postinjection (Fig. 1a). At 720 minutes after endotoxin administration, only one of the six animals showed significant glomerular thrombus formation. The severity of the glomerular thrombosis varied from case to case, but generally more than half of the glomeruli were affected in the thrombotic animals. Large numbers of intraglomerular fibrin thrombi were present in the thrombotic animals, as demonstrated by direct IF with an antibody to fibrinogen (Fig. 1b). The nonthrombotic rats and the nonthrombotic glomeruli in the thrombotic rats had mild to marked endothelial swelling. Thrombus formation was restricted to glomeruli. However, some scattered fibrinogen strands were also detected by IF in cortical tubulointerstitial capillaries, mainly in the thrombotic animals. Although none of the animals demonstrated glomerular thrombosis at 30 or 90 minutes after endotoxin administration, some glomerular fibrinogen strands were observed by IF in almost all animals at 90 minutes. Additionally, there was a slight but significant increase in the number of glomerular neutrophils beginning 30 minutes after endotoxin administration that remained high for the entire observation period. None of the animals showed cortical necrosis, prominent vascular necrosis, or arterial thrombosis.

\section{Electron Microscopy}

Transmission electron microscopy revealed some degree of glomerular endothelial cell damage in the
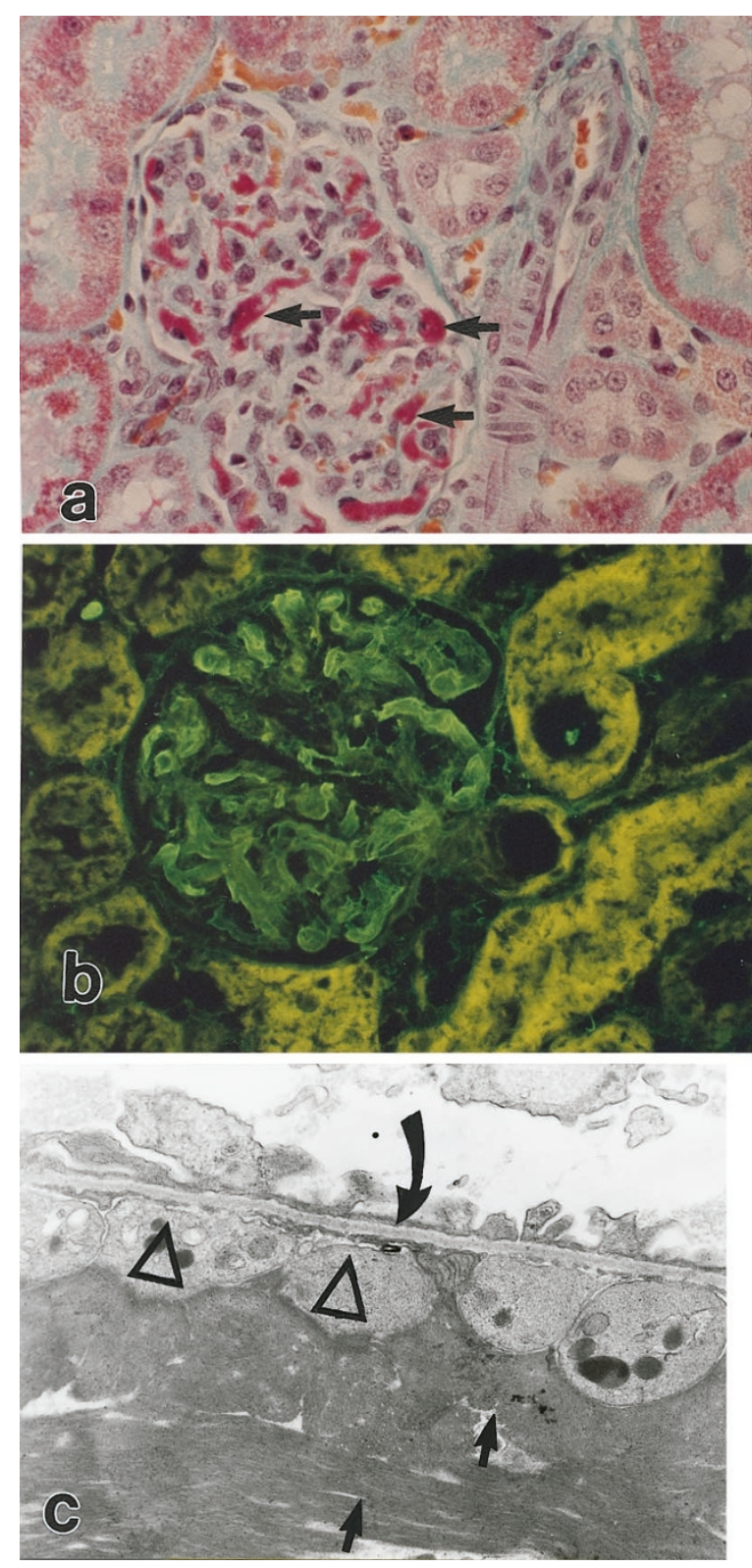

Figure 1.

Glomerular thrombotic microangiography (TMA). (a) A representative glomerulus reveals extensive capillary thrombi (arrows) in a rat killed 180 minutes after endotoxin administration (trichrome stains, original magnification, $\times 400$ ). (b) Direct immunofluorescence with an antibody to fibrinogen shows immunoreactive glomerular intracapillary thrombi in the same animal (original magnification, $\times 400$ ). (c) A representative electron photomicrograph shows a portion of a glomerular capillary loop occluded by electron-dense fibrillary material corresponding to fibrin (arrows). Prominent accumulation of platelets (triangles) along the inner surface of the glomerular capillary endothelium is also seen. Curved arrow indicates the glomerular basement membrane $(x$ $9,000)$.

thrombotic animals at 180, 360, and 720 minutes after endotoxin administration. This damage included moderate focal swelling, mild patchy detachment from the glomerular basement membranes, and increased electron density of the cytoplasm, suggesting necrobiotic changes. The majority of the endothelial cells, however, seemed to be ultrastructurally intact, and maintained characteristic fenestrations. The glomeru- 
lar capillary loops with thrombosis contained electrondense wisps of "fibrillar fibrin" admixed with polymorphonuclear leukocytes and platelets. Some of the polymorphonuclear leukocytes seemed to be adherent to the glomerular endothelium. Polymorphonuclear leukocytes with swelling and disintegration, and occasional free neutrophil granules were also observed. The platelets with various degree of degranulation (activation) seemed to be adherent either to the glomerular endothelium or to the underlying basement membranes (Fig. 1c). Other platelets were embedded in the fibrin.

\section{Plasma Concentration of Nitrite and Nitrate}

Data given in Figure 2 demonstrate that there was a significant increase in the plasma NOx level beginning 180 minutes after endotoxin administration compared with the control group ( $p<0.05$ ). The plasma NOx concentration increased over the course of the experiment.

\section{Immunoperoxidase Studies}

Expression of iNOS in control animals was minimal. There was weak and focal immunoreactivity in occasional glomerular cells and in a few scattered cells in the collecting ducts of the renal cortex and medulla (Fig. 3a). Reactivity to the iNOS antigen 30 and 90 minutes after endotoxin injection was similar to that observed in controls. However, there was a marked increase in iNOS expression in rats 180 minutes after the endotoxin injection that remained elevated during the 12-hour observation period (Fig. 3b and c). iNOS immunoreactivity was seen in glomerular capillary loops, particularly in the circulating neutrophils, monocytes, endothelial cells, and occasional mesangial cells, and also in parietal cells of Bowman's capsule. Additionally, immunoreactivity was found in cortical

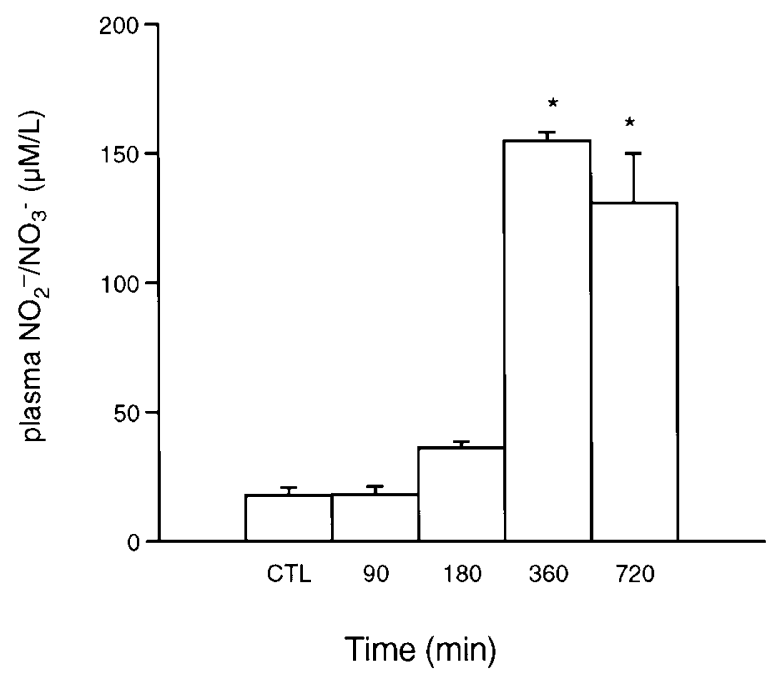

\section{Figure 2.}

Plasma concentration of nitric oxide metabolites (NOX) in control and endotoxin-treated rats during the course of TMA. Values are mean \pm SEM, $n=$ 4 to 6 in each group. ${ }^{\star} p<0.01$ versus control and other time points. peritubular capillaries, collecting ducts, and in scattered interstitial and smooth muscle cells. The medulla was intensely immunoreactive for iNOS in the peritubular vessels, papillary surface epithelium, and, to a lesser degree, in the collecting ducts and thick ascending limbs of Henle.

In control animals, there was weak eNOS immunoreactivity in occasional glomerular endothelial cells, strong immunoreactivity in the endothelium of peritubular capillaries and weaker immunoreactivity in the interlobular arteries and arterioles (Fig. 3d). The rats in the TMA group had a similar distribution pattern, but with a reduced intensity of eNOS 180 minutes after endotoxin administration (Fig 3e). However, the current methodology was not sensitive enough to detect differences over the course of the observation period.

\section{NOS Protein Expression}

The animals exhibited a marked reduction in eNOS protein in the cortical tissue of the kidney beginning 360 minutes after endotoxin administration compared with controls $(p<0.05)$. The eNOS protein mass declined further over the course of the study (Fig. 4). In contrast to eNOS, the iNOS protein content of renal cortical tissue showed a significant rise 360 minutes after endotoxin injection and remained elevated during the entire observation period (Fig. 5).

\section{In Vitro Studies}

Incubation of glomeruli (harvested from control animals) with endotoxin for 6 hours resulted in a marked reduction in eNOS protein expression that declined further by 24 hours. In contrast, glomeruli exposed to endotoxin for 6 hours exhibited a marked increase in iNOS protein content that was further increased by 24 hours of incubation (Fig. 6). Surprisingly, there was no discernible difference in NOx recovered from the incubation media between the endotoxin-treated and control glomeruli at either 6 hours $(270 \pm 30$ versus $260 \pm 30 \mathrm{nM} / \mathrm{mg}$ of protein, $p>0.05)$ or 24 hours (290 \pm 4 versus $270 \pm 4 \mathrm{nM} / \mathrm{mg}$ of protein, $p>0.05)$. The lack of a significant rise in NOx generation in isolated glomeruli incubated with endotoxin contrasts with the rise in plasma NOx in endotoxin-treated animals. The reason for this disparity is not clear. However, it may be due to the abundance of circulating leukocytes, tissue macrophages, and other endotoxin-cytokine susceptible cells (eg, hepatocytes) in intact animals and their paucity in the isolated normal glomeruli. Additionally, limitation of substrate availability in vitro and its abundance in vivo may also contribute to this apparent disparity.

\section{Discussion}

TMA are uncommon clinicopathologic syndromes that are manifested as thrombocytopenia and microangiopathic hemolysis with signs of kidney and brain involvement. It is believed that microvascular endothelial cell injury is central to the pathogenesis of TMA (Laszik and Silva, 1998). NO, otherwise known as 

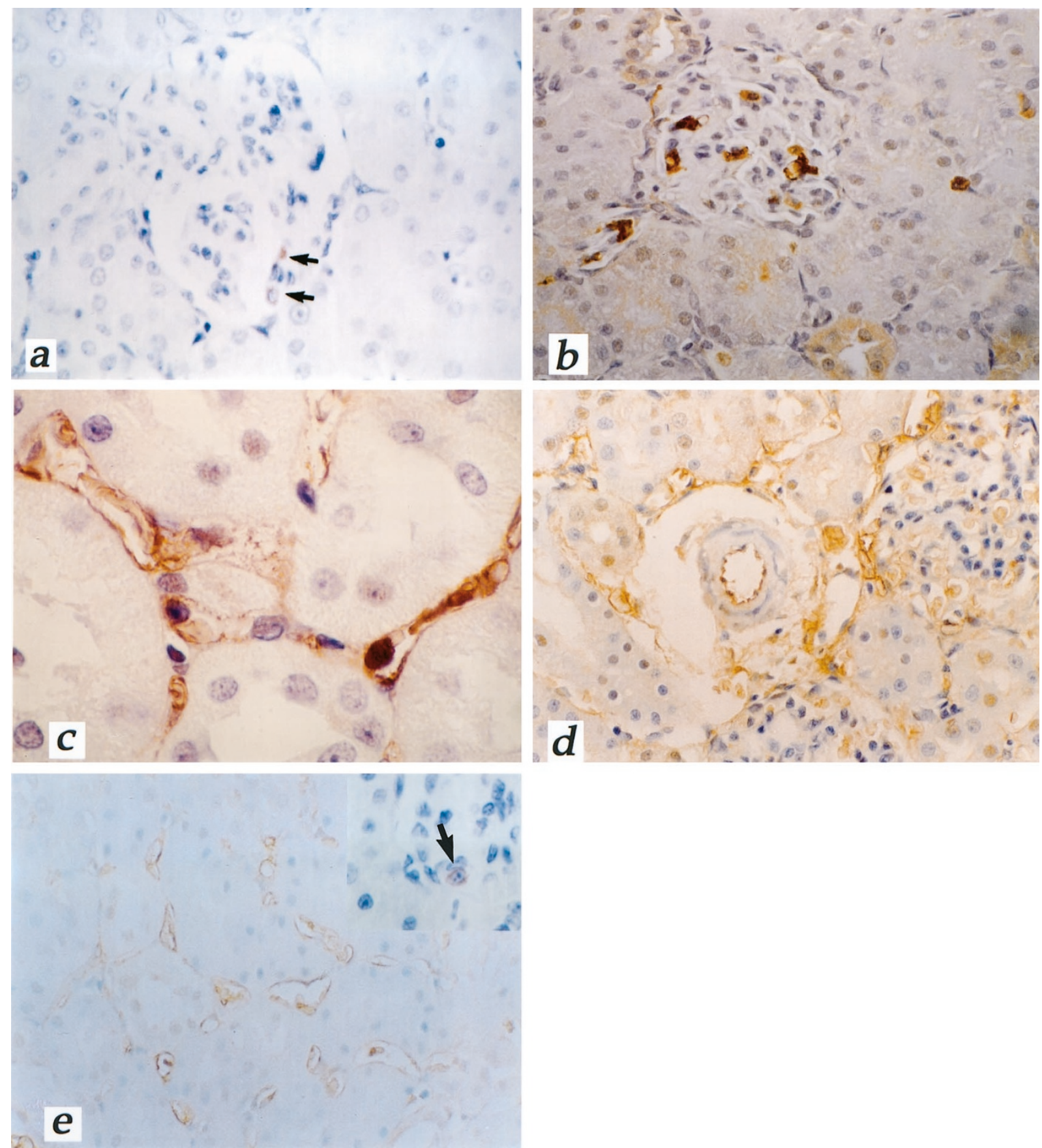

\section{Figure 3.}

Endothelial and inducible nitric oxide synthase (eNOS and iNOS) immunohistochemistry. (a) A representative glomerulus from a normal rat shows occasional immunoreactivity for iNOS in glomerular cells (arrows, original magnification $\times 400)$. (b and c) iNOS immunoreactivity 180 minutes after endotoxin administration reveals intense immunoreactivity in glomerular cells, arterioles, peritubular capillaries, and scattered interstitial cells (b: original magnification, $\times 200$; c: original magnification, $\times 600$ ). (d) Representative photomicrograph from control rats reveals weak eNOS immunoreactivity in occasional glomerular cells and strong immunoreactivity in arterioles and peritubular capillaries (original magnification, $\times 200$ ). (e) Representative photomicrograph from rats 180 minutes after endotoxin injection demonstrates diffuse peritubular capillary and occasional glomerular cell immunoreactivity (inset) for eNOS. Although the intensity of eNOS immunoreactivity is slightly less in the TMA group, the current methodology is not sensitive enough to detect differences in the course of TMA (original magnification, $\times 200$ ).

endothelium-derived relaxing factor or EDRF, is a potent vasodilator and plays an important role in the regulation of renal and systemic vascular resistance and in tissue perfusion. Additionally, NO serves as a potent inhibitor of platelet adhesion/aggregation that prevents platelet thrombus formation. However, the effects of TMA and the associated endothelial injury on NOS expression in the kidney is unknown.

In this study, endotoxin-treated animals developed glomerular capillary thrombosis. This was associated with a significant decline in eNOS protein mass in the renal cortical tissue, which corresponded to reduced 


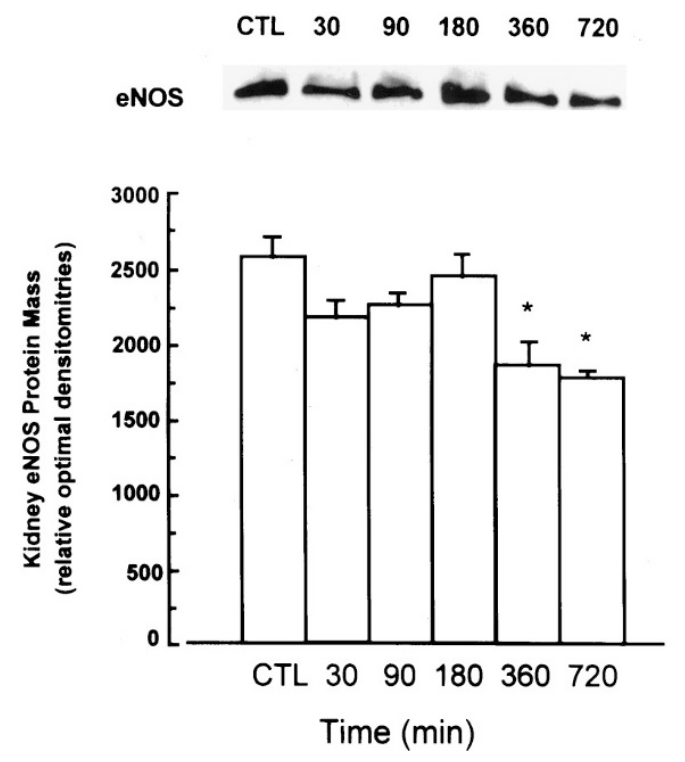

Figure 4.

Representative Western blot of kidney eNOS in a control $(C T L)$ rat and in rats killed at various time points during the course of TMA (top panel). Group data illustrating the relative optical densities of the eNOS protein bands in the study animals. $n=6$ for each group. ${ }^{*} p<0.05$ relative to other groups (bottom panel).

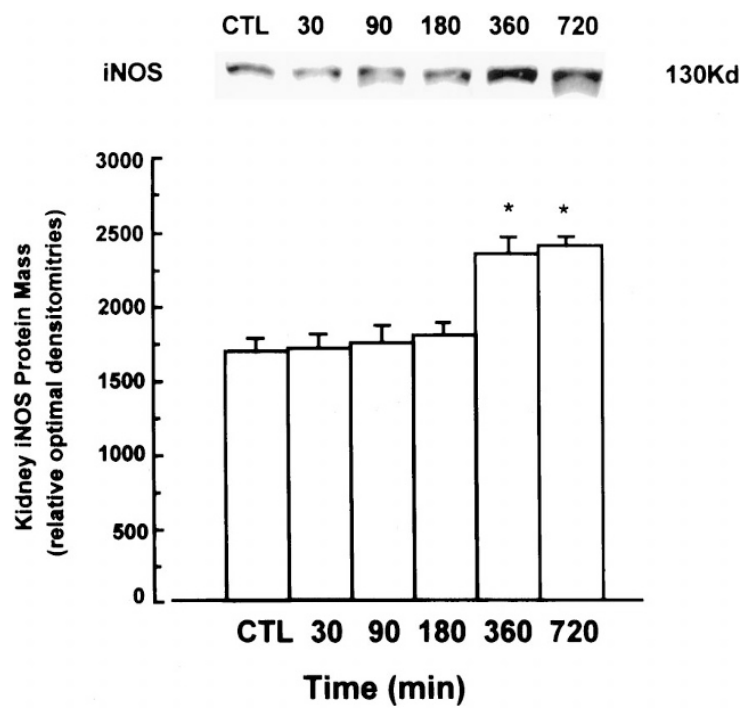

Figure 5.

Representative Western blot of kidney iNOS in a control (CTL) rat and in rats killed at various time points during the course of TMA (top panel). Group data illustrating relative optical densities of the iNOS protein bands in the study animals. $n=6$ for each group. ${ }^{*} p<0.05$ relative to other groups (bottom panel).

eNOS immunoreactivity in endothelial cells of peritubular capillaries, arterioles, interlobular arteries, and scattered glomerular cells. The decline in the eNOS protein is due to endothelial damage or injury, and/or to an increased production of cytokines, such as $\mathrm{TNF} \alpha$, which has been shown to destabilize eNOS mRNA and dramatically reduce eNOS activity in endothelial cells in vitro (Yoshizumi et al, 1993). Additionally, overproduction of NO by iNOS may depress
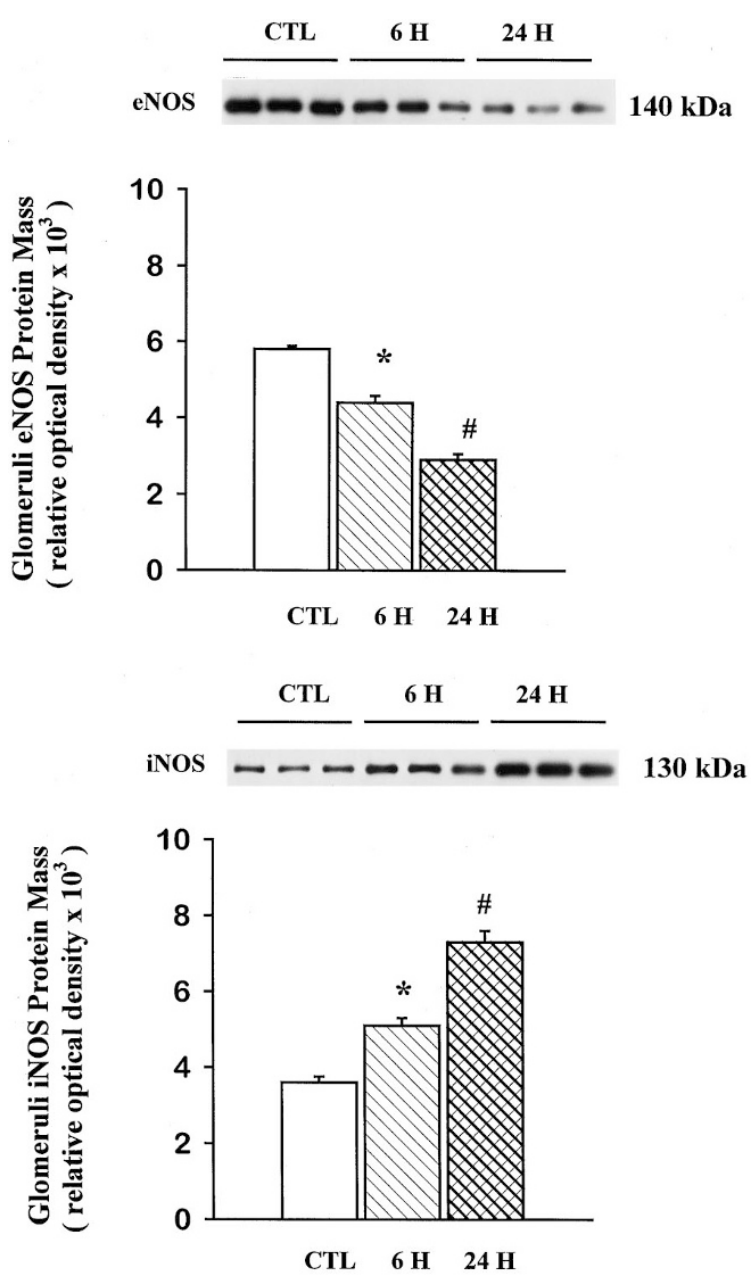

Figure 6.

Representative Western blot and the corresponding group data depicting eNOS (upper panel) and iNOS (lower panel) protein abundance in isolated glomeruli incubated with endotoxin for 6 and 24 hours. $n=6$ parallel experiments. * $p<$ 0.05 versus control; $\# p<0.05$ versus control and 6 hours.

eNOS enzymatic activity by autoinhibition via specific binding sites on the enzyme and downregulate eNOS protein expression (Schwartz et al, 1997; Vaziri and Wang, 1999). In fact, we demonstrated a marked increase in plasma NOx levels starting 180 minutes after endotoxin administration, this coincided with the peak thrombus formation in the glomeruli. Therefore, there is an apparent dissociation between systemic NO production and intrarenal NO availability. We believe that the decline in endothelial NO production in glomerular microenvironment can lead to a vicious cycle of further endothelial vasoconstriction-induced ischemia together with enhanced platelet aggregation, resulting in microthrombus formation. Indeed, the animals treated with endotoxin showed either diffuse microthrombi or early signs of thrombus formation manifested by swelling of the endothelial cells, platelet adhesion, and fibrinogen deposition in the glomerular capillaries beyond 180 minutes after endotoxin administration. These changes were coupled with decreased eNOS expression. To our knowledge, ours is the first 
study demonstrating depressed local renal tissue eNOS expression in the course of TMA.

The alternative mechanism of downregulation of eNOS involves the possible effect of endotoxin on eNOS gene transcription and translation. Schwartz et al (1997) showed that the glomerular eNOS mRNA level was not altered by incubation with endotoxin in vitro. However, they did not explore the effect of endotoxin on eNOS protein expression. In this study, we demonstrated a marked reduction in eNOS protein expression in isolated normal glomeruli incubated with endotoxin. This was associated with a marked upregulation of iNOS protein expression, mirroring the effects seen with endotoxin administration in vivo. These observations imply that the reduction in eNOS protein content was, at least in part, due to the inhibitory effect of either endotoxin or iNOS-derived $\mathrm{NO}$ on eNOS protein expression.

It is of interest that renal cortical tissue iNOS protein mass markedly increased 360 minutes after endotoxin administration. This is not surprising because iNOS is induced by a combination of endotoxin and certain cytokines (eg, TNF $\alpha$, IL-1, and interferons) (Estrada et al, 1992; Lamas et al, 1991). To study the cellular basis of this phenomenon, we employed immunoperoxidase techniques to locate the iNOS isozyme expression. We demonstrated that low levels of iNOS are constitutively expressed in the normal kidney, mainly in glomerular cells and scattered collecting ducts. There was a marked increase in iNOS immunoreactivity beginning 180 minutes after endotoxin injection, which remained elevated during the course of the experimental period. In glomeruli, iNOS immunoreactivity was predominantly found in circulating neutrophils and monocytes and in occasional endothelial and mesangial cells. The medulla showed intense immunoreactivity for iNOS in peritubular capillaries. Additionally, arterioles, collecting ducts, and scattered interstitial cells were immunoreactive for the iNOS isozyme. These observations are in agreement with previous reports (Bachmann and Mundel, 1994; Mohaupt et al, 1994; Morrissey et al, 1994; Star, 1997; Stuehr and Marletta, 1985).

The marked increase in iNOS expression found in our rats 360 minutes after endotoxin administration can account for the dramatic increase in total body $\mathrm{NO}$ production in the late phase of TMA, as demonstrated by marked increase in plasma NOx concentration. These data support the previous observations by other investigators who showed increased total body NO production in TMA (Herlitz et al, 1997; Noris et al, 1996; Shultz and Raij, 1992; Westberg et al, 1994). It should be noted that increased total body NO derived from iNOS is frequently accompanied by a concomitant release of free radicals, which avidly inactivate NO. Moreover, the availability of iNOS-derived NO to endothelium and platelets is uncertain (Herlitz et al, 1997). Thus, the increase in total body NO production associated with iNOS induction may not be renoprotective.

In vitro incubation of glomeruli with endotoxin showed results concordant with those observed in vivo. The upregulation of iNOS coincided with the downregulation of eNOS, hence leading to no discernible changes of recoverable $\mathrm{NO}$ metabolites in the media. It should be noted that the normality of $\mathrm{NO}$ metabolite recovered does not necessarily imply normal NO availability. This is because induction of iNOS in macrophages, neutrophils, and mesangial cells is coupled with the production and release of various reactive species including reactive oxygen species such as superoxide and hydrogen peroxide (Thiemermann, 1997). These agents are known to react avidly with and inactivate NO, producing reactive nitrogen species and nitrated compounds, which ultimately convert to nitrate and nitrite through isomerization and turnover. Thus, under this condition, the amount of NO metabolite recovered in the media may not reflect the amount of biologically available NO.

The cause and effect relationship between changes in the NO system and the associated microthrombosis is not clear. However, activation of macrophages, neutrophils, and mesangial cells, which is expected to lead to increased reactive oxygen species generation and $\mathrm{NO}$ inactivation, may contribute to thrombosis by diminishing NO availability and thereby increasing platelet adhesion and aggregation. On the other hand, microthrombus formation in the glomerular capillaries can lead to endothelial cell injury or death and hence, depressed eNOS-derived NO production.

In conclusion, experimental TMA resulted in a marked reduction of renal tissue eNOS expression. This can potentially exacerbate renal vasoconstriction and tissue ischemia by decreasing intrarenal endothelial NO production. Additionally, depressed endothelial $\mathrm{NO}$ availability can worsen thrombotic microangiopathy by facilitating platelet adhesion to endothelial surface. The fall in eNOS expression was accompanied by a significant rise in iNOS protein expression that can be explained by the eventual induction of this isozyme by a combination of endotoxin and various cytokines. The rise in NO production through iNOS induction may not be necessarily renoprotective at this stage and may be associated with cytotoxicity and further tissue destruction.

\section{Materials and Methods}

\section{Animals}

Thirty-six male Sprague-Dawley rats (Harlan Sprague Dawley, Indianapolis, Indiana), each weighing approximately $300 \mathrm{~g}$ were housed in a temperaturecontrolled and light-regulated room (12 hours light/12 hours dark cycle) for one week prior to use. Food and water were available ad libitum.

\section{Experimental Procedures}

Procedures were performed on animals under isoflurane anesthesia. A Silastic indwelling catheter was implanted into the right jugular vein and the free end of the catheter exteriorized at the base of the neck. After recovering from anesthesia, the animals were given $E$. coli endotoxin (0127:B8; Sigma, St. Louis, Missouri), 
$20 \mathrm{mg} / \mathrm{kg}$ of body weight, in an iv bolus injection. Subgroups of six animals each were sacrificed before injection and at 30,90, 180, 360, and 720 minutes after injection. This conscious septic rat model has been extensively characterized in previous studies and avoids any effect of anesthesia on the resultant changes (Laszik et al, 1994a, 1994b).

\section{Routine Histology and IF Microscopy}

Formalin-fixed renal tissues from all study groups were processed, paraffin-sectioned, and stained with hematoxylin and eosin, periodic acid Schiff, and trichrome (Laszik et al, 1994a, 1994b).

For immunofluorescence studies, 5- $\mu \mathrm{m}$ thick cryostat sections of the kidneys obtained from all rats were fixed for 10 minutes in cold acetone and air-dried. Fibrinogen was detected in a direct system using fluorescein isothiocyanate-conjugated goat anti-rat fibrinogen antibody (Nordic, Capistrano Beach, California). The degree of glomerular TMA at 180 minutes was characterized by the percentage of thrombotic versus nonthrombotic glomeruli by counting 200 glomeruli on fibrinogen-stained frozen sections in the thrombotic animals (Laszik et al, 1994a, 1994b).

\section{Immunoperoxidase Studies}

Immunohistochemistry for eNOS and iNOS was performed using a standardized streptavidin-biotin-peroxidase method on formalin-fixed paraffin-embedded renal tissues. Except for the incubation with the primary antibodies, all incubations were at room temperature and were separated by washes with PBS. After deparaffinization, sections were treated with $1.25 \%$ hydrogen peroxidase to block endogenous peroxidase activity. After preincubation with $10 \%$ normal horse or swine serum for 20 minutes, sections were incubated with primary antibodies overnight at $4^{\circ} \mathrm{C}$, followed sequentially with biotinylated horse antimouse (Vector Laboratories, Burlingame, California) or biotinylated swine anti-rabbit (DAKO, Carpinteria, California) antibodies for 20 minutes, and streptavidinperoxidase complex (DAKO) for 30 minutes. The working concentrations for the mouse anti-eNOS monoclonal antibody (Transduction Laboratories, Lexington, Kentucky), and rabbit anti-iNOS antibody (Transduction Laboratories) were $0.05 \mu \mathrm{g} / \mathrm{ml}$ and $0.025 \mu \mathrm{g} / \mathrm{ml}$, respectively. For negative controls, a monoclonal mouse IgG1 (Bethyl Laboratories Inc., Montgomery, Texas) or normal rabbit serum was used at equivalent concentrations. Diaminobenzidine (Sigma) was used as the chromogen and hematoxylin was used for a nuclear counterstain.

\section{Electron Microscopy}

In some selected cases (two animals at each time point), transmission electron microscopic examinations were also performed. The $2 \%$ glutaraldehydefixed kidneys were embedded in Epon 812 (Serva, Heidelberg, Germany). The sections were stained with uranyl acetate and lead citrate, and examined in a Hithachi HS9 electron microscope.

\section{NOx Assay}

Plasma and culture media NOx were determined as previously described (Vaziri, 1998) using the purge system of a Sievers Instruments Model 270B Nitric Oxide Analyzer (NOA, Sievers Instruments, Boulder, Colorado).

\section{Tissue Preparation and Western Blot Analysis}

Rats were sacrificed by decapitation. One kidney from each rat was immediately excised, cleaned with PBS, frozen in liquid nitrogen, and stored at $-75^{\circ} \mathrm{C}$. Tissue was homogenized in five volumes of lysis buffer $(1 \%$ SDS, $1.0 \mathrm{mM}$ sodium vanadate, $10 \mathrm{mM}$ Tris $\mathrm{HCl}, \mathrm{pH}$ 7.4) containing $10 \mu \mathrm{M}$ pepstatin, $13 \mu \mathrm{M}$ leupeptin, and $1 \mathrm{~mm}$ phenylmethylsulfonyl fluoride. Homogenates were centrifuged at $12,000 \times g$ for 5 minutes at $4^{\circ} \mathrm{C}$ to remove tissue debris without precipitating plasma membrane fragments (Vaziri et al, 1998). Protein concentration was determined by using a Bio-Rad kit (Bio-Rad Laboratories, Hercules, California).

Western blot analysis was carried out to determine the endothelial and inducible NOS (eNOS and iNOS) protein masses as previously described (Vaziri et al, 1998). The eNOS monoclonal antibody, peroxidaseconjugated goat anti-mouse IgG antibody, anti-Mac NOS-I, human endothelial positive control, and mouse macrophage positive control were supplied by Transduction Laboratories. Briefly, kidney tissue preparations were size-fractionated on $4 \%$ to $12 \%$ TrisGlycine gel (Novex, San Diego, California) at $120 \mathrm{~V}$ for 3 hours. In preliminary experiments, we found that the given protein concentrations were within the linear range of detection for our Western blot technique. After electrophoresis, proteins were transferred onto hybond-ECL membrane (Amersham Life Science Inc., Arlington Heights, Illinois) at $400 \mathrm{~mA}$ for 2 hours using the Novex transfer system. The membrane was prehybridized in $10 \mathrm{ml}$ of buffer A (10 mM Tris hydrochloride, $\mathrm{pH} 7.5,100 \mathrm{mM} \mathrm{NaCl}, 0.1 \%$ Tween 20, and 10\% nonfat milk powder) for 1 hour and then hybridized for an additional hour in the same buffer with $10 \mu \mathrm{l}$ of the given monoclonal antibody to NOS (1:1000 dilution). The membrane was then washed for 30 minutes in a shaking bath, changing the wash buffer (buffer $A$ without nonfat milk) every 5 minutes, and incubated for 1 hour in buffer $A$ with a 1:1000 dilution of goat anti-mouse IgG-horse radish peroxidase. Experiments were carried out at room temperature. The washes were repeated before the membrane was developed with a light-emitting nonradioactive method using ECL reagent (Amersham Life Science Inc.). The membrane was subjected to autoluminography for 1 to 5 minutes. The autoluminographs were scanned with a laser densitometer (Model PD1211, Molecular Dynamics, Sunnyvale, California) to determine the relative optical densities of the bands. In all instances, the membranes were stained with Ponceau stain, which veri- 
fied the uniformity of protein load and transfer efficiency across the test samples.

\section{In Vitro Studies}

Additional normal control rats were used for these studies. Kidneys were decapsulated and bisected sagittally. After removing the medulla, the cortex was washed twice with PBS. Glomeruli were prepared using a sieving technique described previously (Pahl et al, 1996). Briefly, renal cortices were minced and successively passed through 40 to 150 mesh sieves. The glomeruli were collected on a 200 mesh sieve, washed 3 to 4 times with ice-cold PBS, resuspended in RPMI 1640 medium (Sigma). The suspension was aliquoted and divided into control and endotoxin treated groups. The glomeruli in a treatment group were incubated with $1 \mu \mathrm{g} / \mathrm{ml}$ of endotoxin for 6 and 24 hours, respectively. At the end of incubation, the glomeruli were harvested with lysis buffer and homogenized. Western blot analysis was performed as described earlier. Additionally, the culture media was collected for the measurement of NOx.

\section{Data Presentation and Analysis}

Data are presented as means $\pm \mathrm{SE}$. ANOVA was used for data analysis. $p \leq 0.05$ was considered statistically significant.

\section{References}

Bachmann S and Mundel P (1994). Nitric oxide in the kidney: Synthesis, localization and function. Am J Kidney Dis 24: 112-129.

Bertani T, Abbate M, Zoja C, Corna C, Perico N, Ghezzi P, and Remuzzi G (1989). Tumor necrosis factor induces glomerular damage in the rabbits. Am J Pathol 134:419-430.

Bevilacqua MP, Pober JS, Majeau GR, Fiers W, Cotran RS, and Gimbrone MA (1986). Recombinant tumor necrosis factor induces procoagulant activity in cultured human vascular endothelium: Characterization and comparison with the actions of interleukin-1. Proc Natl Acad Sci USA 83:4533-4537.

Cybulsky MI, Chan MKW, and Movat HZ (1988). Biology of disease. Acute inflammation and microthrombosis induced by endotoxin, interleukin-1, and tumor necrosis factor and their implications in gram negative infection. Lab Invest 58:365-378.

Estrada C, Gomez C, Martin C, Moncada S, and Gonzalez C (1992). Nitric oxide mediates tumor necrosis factor- $\alpha$ cytotoxicity in endothelial cells. Biochem Biophys Res Commun 186:475-482.

Herlitz $H$, Petersson A, Sigström L, Wennmalm Å, and Westberg G (1997). The arginine-nitric oxide pathway in thrombotic microangiopathy. Scand J Urol Nephrol 31:477479.

Lamas S, Michel T, Brenner BM, and Marsden PA (1991). Nitric oxide synthesis in endothelial cells: Evidence of a pathway inducible by TNF $\alpha$. Am J Physiol 261:C634-C641.

Laszik Z, Carson WC, Nadasdy T, Johnson LD, Lerner MR, Brackett DJ, Esmon CT, and Silva FG (1994a). Lack of suppressed renal thrombomodulin expression in a septic rat model with glomerular thrombotic microangiopathy. Lab Invest 70:862-867.

Laszik Z, Nadasdy T, Johnson LD, Lerner MR, Brackett D, and Silva FG (1994b). Renal interleukin-1 expression during endotoxemia and gram-negative septicemia in conscious rats. Circulat Shock 43:115-121.

Laszik Z and Silva FG (1998). Thrombotic microangiopathy. In: Jennette JC, Olson JL, Schwartz M, and Silva FG, editors. Heptinstall's Pathology of the Kidney. Philadelphia: Lippincot-Raven, 1003-1057.

Marletta MA (1989). Nitric oxide: Biosynthesis and biological significance. Trends Biochem Sci 14:488-492.

Mohaupt MG, Elzie JL, Ahn KY, Clapp WL, Wilcox CS, and Kone BC (1994). Differential expression and induction of mRNAs encoding two inducible nitric oxide synthases in rat kidney. Kidney Int 46:653-65.

Moncada S and Higgs EA (1995). Molecular mechanism and therapeutic strategies related to nitric oxide. FASEB J 9:1319-1330.

Moncada S, Palmer RMJ, and Higgs EA (1991). Nitric oxide: Physiology, pathophysiology, and pharmacology. Pharmacol Rev 43:109-142.

Morrissey JJ, McCracken R, Kaneto H, Vehaskari M, Montani D, and Klahr S (1994). Location of an inducible nitric oxide synthase mRNA in the normal kidney. Kidney Int 45:9981005.

Nawroth PP, Handley DA, Esmon CT, and Stern DM (1986). Interleukin 1 induces endothelial cell procoagulant while suppressing cell surface anticoagulant activity. Proc Natl Acad Sci USA 83:3460-3464.

Nawroth PP and Stern DM (1986). Modulation of endothelial cell hemostatic properties by tumor necrosis factor. J Exp Med 163:740-745.

Noris M, Ruggenenti P, Todeschini M, Figliuzzi M, Macconi D, Zoja C, Paris S, Gaspari F, and Remuzzi G (1996). Increased nitric oxide formation in recurrent thrombotic microangiopathies: A possible mediator of microvascular injury. Am J Kidney Dis 27:790-796.

Pahl MV, Wang J, Zhou XJ, Wang XQ, Tehranzadeh A, and Vaziri ND (1996). Effect of low density lipoprotein on cytosolic $\left[\mathrm{Ca}^{2+}\right]$ in cultured rat mesangial cells. J Invest Med 44:556560 .

Pober JS and Cotran RS (1991). What can be learned from the expression of endothelial adhesion molecules in tissues? Lab Invest 64:301-305.

Radomski MW, Palmer RMJ, and Moncada S (1987). Endogenous nitric oxide inhibits human platelet adhesion to vascular endothelium. Lancet 2:1057-1058.

Radomski MW, Vallance P, Whitley G, Foxwell N, and Moncada S (1993). Platelet adhesion to human vascular endothelium is modulated by constitutive and cytokine induced nitric oxide. Cardiovasc Res 27:1380-1382.

Raij L (1994). Glomerular thrombosis in pregnancy: Role of the L-arginine-nitric oxide pathway. Kidney Int 45:775-781.

Schwartz D, Mendonca M, Schwartz I, Satriano J, Wilson CB, and Blantz RC (1997). Inhibition of constitutive nitric oxide synthase (NOS) by nitric oxide generated by inducible NOS after lipopolysaccharide administration provokes renal dysfunction in rats. J Clin Invest 100:439-448. 
Shultz PJ and Raij L (1992). Endogenously synthesized nitric oxide prevents endotoxin-induced glomerular thrombosis. $\mathrm{J}$ Clin Invest 90:1718-1725.

Star RA (1997). Intrarenal localization of nitric oxide synthase isoforms and soluble guanylyl cyclase. Clin Exp Pharmacol Physiol 24:607-610.

Stuehr DJ and Marletta MA (1985). Mammalian biosynthesis: Mouse macrophages produce nitrite and nitrate in response to Escherichia coli lipopolysaccharide. Proc Natl Acad Sci USA 82:7738-7742.

Thiemermann C (1997). Nitric oxide and septic shock. Gen Pharmacol 29:159-166.

Vaziri ND, Ni Z, Wang XQ, Oveisi F, and Zhou XJ (1998). Downregulation of nitric oxide synthase in chronic renal insufficiency: Role of excess PTH. Am J Physiol 274:F642F649.
Vaziri ND and Wang XQ (1999). cGMP-mediated negative feedback regulation of endothelial nitric oxide synthase by nitric oxide. Hypertension 34:1237-1241.

Wakabayashi G, Gelfand JA, Burke JF, Thompson RC, and Dinarello CA (1991). A specific receptor antagonist for interleukin 1 prevents Escherichia coli-induced shock in rabbits. FASEB J 5:338-343.

Westberg G, Shultz PJ, and Raij L (1994). Exogenous nitric oxide prevents endotoxin-induced glomerular thrombosis in rats. Kidney Int 46:711-716.

Yoshizumi M, Perrella MA, Burnett SC, and Lee ME (1993). Tumor necrosis factor downregulates an endothelial nitric oxide synthase mRNA by shortening its half-life. Cir Res 73:205-209. 